\title{
Die baltischen Staaten und die EU: der steinige Weg von ,außerhalb' zum ,Kern'
}

\author{
Andres Kasekamp*
}

Die baltischen Staaten Estland, Lettland und Litauen sind die einzigen Länder der ehemaligen Sowjetunion, die der Europäischen Union beigetreten sind. Dieser Erfolg beruht darauf, dass ihre Zugehörigkeit zu Europa nicht nur eine geopolitische Wahl war, sondern grundsätzlich im Einklang mit ihren Werten und ihrer Identität steht. In den vergangenen zwei Jahrzehnten überwanden sie erfolgreich zwei Umbrüche: den mühevollen Übergang von einer Planwirtschaft zur freien Marktwirtschaft nach dem Zusammenbruch der Sowjetunion und die drastischen Sparmaßnahmen, die den Ländern zu Beginn der aktuellen globalen Finanzkrise auferlegt wurden.

Obwohl Estland, Lettland und Litauen oft als die ,baltischen Staaten` zusammen ,in einen Topf geworfen' werden, ist dieser Begriff relativ neu und seine Bedeutung hat sich im Laufe der Zeit verändert. Ein Beispiel für nationale Unterschiede zwischen den drei Staaten sind ihre Sprachen: Litauisch und Lettisch bilden den baltischen Zweig der indoeuropäischen Sprachfamilie, Estnisch hingegen ist eng mit Finnisch verwandt und gehört zur finno-ugrischen Sprachfamilie. Dennoch haben die Letten und Esten historisch und kulturell viel mehr gemeinsam als mit den Litauern. Darüber hinaus sind Letten und Esten überwiegend Lutheraner, während die Litauer Katholiken sind.

Der Beitrag gliedert sich in drei Teile: Der erste Teil erklärt den historischen Hintergrund sowie die Gründe und Wege, die Estland, Lettland und Litauen in die Europäische Union geführt haben. Der zweite Teil befasst sich mit der heutigen Europapolitik in den baltischen Staaten und deren Rolle in der Europäischen Union. Der letzte Teil wagt einen Ausblick in die Zukunft der baltischen Staaten und deren Prioritäten in der Europäischen Union.

\section{Rückkehr nach Europa}

\section{Geschichtlicher Hintergrund: gefährdete Unabhängigkeit}

Bis zum 20. Jahrhundert waren die baltischen Nationen recht verschieden und wurden nicht als zusammengehörig angesehen. Estland und Lettland wurden im 13. Jahrhundert von deutschen Ordensrittern unterjocht. Eine kleine deutsche Minderheit blieb dort trotz dänischer, polnischer, schwedischer und schließlich russischer Herrschaft bis 1917 die dominierende politische, soziale und wirtschaftliche Elite. Litauen wiederum wurde im 15. Jahrhundert der größte Staat in Europa und kontrollierte einen Großteil der Gebiete des heutigen Weißrussland und der westlichen Ukraine. Der Unabhängigkeit des Großherzogtums Litauen nahm nach der Union mit Polen im Jahre 1569 kontinuierlich ab bis zur Absorption durch das russische Reich im Jahr 1795.

\footnotetext{
* Prof. Dr. Andres Kasekamp, Professor für baltische Politik an der University of Tartu; Direktor des Estonian Foreign Policy Institute, Tallinn.

Eine frühere Fassung des Beitrags in englischer Sprache ist erschienen in Agnia Grigas/Andres Kasekamp/Kristina Maslauskaite/Liva Zorgenfreija: The Baltic States in the EU: Yesterday, Today and Tomorrow, Notre Europe: Studies \& Reports Nr. 98, Juli 2013, S. 16-32.
} 
Im 20. Jahrhundert erfuhren die Esten, Letten und Litauer ein nationales Wiedererstarken. Nach dem Ende des ersten Weltkriegs erkämpften sie sich erfolgreich ihre Unabhängigkeit von Russland. Gleichwohl konnten sie ihre Staatlichkeit nur bis zum Hitler-Stalin-Pakt von 1939 genießen, welcher Osteuropa in Einflusssphären zerstückelte. Litauen, Lettland und Estland wurden zeitgleich militärisch besetzt und 1940 von der Sowjetunion annektiert. Einem Jahr sowjetischen Terrors folgte die nationalsozialistische Besatzung, während derer die jüdische Bevölkerung im Holocaust ermordet wurde. Im Jahr 1944 kehrte die Rote Armee zurück, der mit jahrelangem bewaffnetem Widerstand begegnet wurde, besonders intensiv war dieser in Litauen. Der Widerstand wurde jedoch durch die Kollektivierung der Landwirtschaft sowie durch Massendeportationen im Jahr 1949 gebrochen. Das gravierendste Erbe aus der Sowjetzeit waren die drastischen demografischen Verschiebungen in Estland und Lettland. Ethnisch war die Bevölkerung Estlands im Jahr 1945 zu mehr als 90 Prozent estnisch und jene in Lettland zu fast 80 Prozent lettisch. Nach dem Ende der sowjetischen Zeit war der Anteil der Esten auf 62 Prozent und der Anteil der Letten auf nur 52 Prozent gesunken, was den Fortbestand einer ethnischen Mehrheit in den jeweiligen Heimatländern infrage stellte. Der Wunsch, diesen negativen Trend aufzuhalten, wurde einer der treibenden Faktoren der Unabhängigkeitsbewegung in den späten 1980er Jahren. ${ }^{1}$

Die 1986 beginnende Glasnost- und Perestroika-Ära des sowjetischen Staatschefs Michail Gorbatschow nutzten die Letten, Esten und Litauer als Chance, ihren Wunsch nach Freiheit auszudrücken. Dies kumulierte in der friedlich verlaufenden baltischen „Singenden Revolution“2 im Jahr 1988. Die ersten demokratisch gewählten Regierungen in Estland, Lettland und Litauen gaben im Jahr 1990 ihren Willen bekannt, die Unabhängigkeit der Staaten wiederherzustellen. Dieser Schritt konnte schließlich während des gescheiterten Putsches in Moskau im August 1991 realisiert werden. Da die westlichen Staaten die sowjetische Annexion der baltischen Staaten im Jahr 1940 de jure nie anerkannt hatten, verlief die erneute Aufnahme der baltischen Staaten in die internationale Gemeinschaft reibungslos.

\section{Der Weg zur EU-Mitgliedschaft: eine von drei Optionen}

Nach der Wiederherstellung ihrer Unabhängigkeit im August 1991 hatten Estland, Lettland und Litauen drei Möglichkeiten für ihre geopolitische Orientierung: Re-Integration in den Osten, Integration in den Westen oder Neutralität. Neutralität nach finnischem Beispiel, unter Sicherstellung der Unabhängigkeit und gleichzeitiger Beibehaltung der engen Beziehungen zum Osten, schien die realistischste Option zu sein und wurde von ,Freunden“ aus der internationalen Gemeinschaft empfohlen. Diese Option passte auch zu dem populären Slogan der Zeit, eine ,Brücke zwischen Ost und West' zu sein. Auch die Re-Integration in den Osten war damals als Option nicht so unrealistisch, wie es aus heutiger Sicht erscheint, da sich Boris Jelzins Russland der frühen 1990er Jahre in Richtung Demokratie und freie Marktwirtschaft bewegte. Russische Demokraten waren Verbündete der Balten im Kampf gegen das kommunistische Regime. Darüber hinaus blieben Russland und die Gemeinschaft Unabhängiger Staaten zunächst die wichtigsten Handelspartner der Balten.

Die Integration mit Westeuropa erschien am wenigsten wahrscheinlich und damals ein utopisches Szenario zu sein. Historisch gesehen sprach jedoch Einiges gegen die anderen beiden Optionen: Erstens hatten die baltischen Staaten 1939 zwar ihre Neutralität erklärt, dies rettete sie jedoch nicht vor der Besetzung durch die Sowjetunion und Nazi-Deutschland.

1 Andres Kasekamp: A History of the Baltic States, Basingstoke 2010.

2 Das Singen verbotener Volkslieder war zu Sowjetzeiten ein Ausdruck politischen Protests. 
Die Lektion der Geschichte zeigte, dass es die baltischen Staaten vermeiden sollten, isoliert und ohne starke Verbündete zu sein. Zweitens trieb die katastrophale Erfahrung von fast einem halben Jahrhundert sowjetischer Herrschaft die Balten gen Westen. Sie waren fest entschlossen, sich so schnell und so weit wie möglich von Russland und der damit verbundenen Politik zu entfernen. Drittens versank das post-sowjetische Russland im Chaos, während Westeuropa die Hoffnung auf Freiheit und Wohlstand bot. Schließlich gab es ein allgemeines Gefühl von einer ,Rückkehr nach Europa' und damit die Chance einer Wiederherstellung der Werte und Verbindungen, an denen Esten, Letten und Litauer vor der Katastrophe des Zweiten Weltkriegs bereits teilhatten.

Die Strategie der Balten war, möglichst vielen internationalen und westlichen Organisationen beizutreten und das so schnell wie möglich. Indem sie sich in ein dichtes Netzwerk internationaler Gremien einbanden, konnten sie den Fortbestand ihrer Unabhängigkeit sichern. In dieser Entschlossenheit waren die politischen Entscheidungsträger von einem Gefühl der Dringlichkeit getrieben: Ein Zeitfenster war geöffnet, das genutzt werden musste, solange Russland schwach war. ${ }^{3}$ Auch heute noch ist diese Logik Grundlage für politische Entscheidungen, wie zum Beispiel jene Estlands und Litauens, der Eurozone beizutreten, zeigen.

$\mathrm{Zu}$ den wichtigsten Meilensteinen auf dem Weg zur Konsolidierung der Souveränität und Integration der baltischen Staaten in den Westen gehören die Beitritte zum Europarat zwischen 1993 und 1995, der Abzug der russischen Truppen in den Jahren 1993 und 1994 und die 1995 geschlossenen Europa-Abkommen. Der Beitritt Finnlands, Schwedens und Österreichs zur Europäischen Union am 1. Januar 1995 gab Estland, Lettland und Litauen Anlass, selbst Beitrittsgesuche zu stellen. Finnland und Schweden waren in der Zwischenzeit die wichtigsten Handelspartner der baltischen Staaten geworden, insbesondere für Estland. Deren EU-Beitritt bedeutete eine grundlegende geostrategische Neuordnung im Norden Europas. So war es kein Zufall, dass Estland, Lettland und Litauen zwar unabhängig voneinander, aber innerhalb nur weniger Monate in diesem Jahr offiziell ein Beitrittsgesuch stellten.

Wie bei fast jeder EU-Erweiterung, begannen die damaligen neuen Mitglieder sich für ihre Nachbarländer einzusetzen, um die Zone der Stabilität und des Wohlstands um sie herum zu erweitern. Zur Überraschung vieler war Estland unter den fünf mittel-osteuropäischen Ländern das erste Land, welches eingeladen wurde, die Beitrittsverhandlungen im Jahr 1997 formell zu beginnen. Estland wurde vorgezogen, weil es seine marktwirtschaftlichen Reformen schneller vorangetrieben hatte. Zwar waren Lettland und Litauen zunächst darüber verärgert, dass ihre Gesuche zurückgestellt wurden. Der Erfolg Estlands gab ihnen aber die Motivation, ihre Anstrengungen zu verdoppeln. Ihre Bemühungen wurden alsbald belohnt und die Beitrittsverhandlungen weniger als zwei Jahre später eröffnet. Anfänglich sah es so aus, als würde die Europäische Union den ,Regatta-Ansatz' in der Erweiterung verfolgen, das heißt jeder Kandidat würde auf Basis seiner eigenen Leistungen die Verhandlungen individuell beenden und der Europäischen Union beitreten. Etsland, insbesondere dessen Außenminister Toomas Hendrik Ilves, begann daher die ,nordische“ Identität des Landes zu betonen. Der Hauptbeweggrund dafür war, Estland von der , baltischen` Identität abzusetzen, um so individuell und nicht als Teil der baltischen Staaten behandelt zu werden. Die Angst war, dass, wenn unter den baltischen Staaten nicht differenziert würde, sich der Beitritt Estlands verzögern könnte bis der langsamste Reformer der drei baltischen Staaten - Litauen -

3 Piret Ehin: Estonia: Excelling at Self-Exertion, in: Simon Bulmer/Christian Lequesne (Hrsg.): The Member States of the European Union, Oxford 2013, hier S. 214. 
bereit für den Beitritt war. ${ }^{4}$ Ironischer Weise verfolgte Litauen zur gleichen Zeit einen ähnlichen Ansatz hinsichtlich der NATO-Mitgliedschaft. ${ }^{5}$ Es hatte unter den drei baltischen Staaten die stärkste Position inne und versuchte seine Identität von ,baltisch' auf , mittelosteuropäisch` umzumünzen.

\section{Der EU-Beitrittsprozess}

Ein bedeutendes Merkmal der Erweiterung im Jahr 2004 stellte die Gewichtung objektiver Kriterien dar, das heißt der Kopenhagener Kriterien, was den Bestrebungen der baltischen Staaten dienlich war. Im Gegensatz zu Polen war der EU-Beitritt für die baltischen Staaten nicht nur eine Frage des ,wann', sondern auch eine Frage des ,ob'. Sie hatten eine schwächere Ausgangsposition als die anderen mittelosteuropäischen Staaten: Sie waren die ärmsten und am wenigsten bekannten Anwärter, ihre Grenze zur ehemaligen Sowjetunion blieb eine geopolitisch ,rote Linie“ und es gab die Befürchtung, dass Russland verärgert werden könnte und sich in den Umgang mit den russischsprachigen Minderheiten in Estland und Lettland einmischen würde. Politisch gesehen, waren die baltischen Staaten am angreifbarsten und ein Zurücklassen der Länder im Beitrittsprozess nicht gänzlich auszuschließen. Ihre großen Fortschritte bei der Erfüllung der ,objektiven' Kriterien beugte diesem Szenario jedoch vor.

Anders als die ehemaligen Staaten des Warschauer Paktes, ${ }^{6}$ die zwar unter kommunistischer Herrschaft gewesen waren, aber ihre Souveränität behalten hatten, mussten die baltischen Staaten ganz von vorn beginnen. Sie hatten keine Armee, kein Verteidigungsministerium, keine Diplomaten, keine Landeswährung, keine Zentralbank, keinen Grenzschutz, keine Zollbehörden etc. Einerseits war dies ein großer Nachteil, andererseits ermöglichte es, sich an ,best practise"-Lösungen aus der Europäischen Union zu orientieren. Tatsächlich kann es manchmal schwieriger sein, eingefahrene Bürokratien zu reformieren als unerfahrene, jedoch hoch motivierte junge Beamte auszubilden, die offen für eine radikale Änderung des Systems sind.

Die EU-Beitrittsverhandlungen verliefen relativ reibungslos, aber einige Schwierigkeiten gab es dennoch.

Erstens stellte die Stilllegung des Kernkraftwerks Ignalina in Litauen das wohl komplexeste Problem dar. ${ }^{7}$ Zweitens versuchten die meisten mittelosteuropäischen Staaten Ausnahmeregelungen hinsichtlich kostenintensiver Standards im Umwelt- und Pflanzenschutzbereich zu erreichen. Drittens war eine Reformierung der Justizsysteme und des Strafvollzugs erforderlich. Viertens hatten die kleinen, jungen Staaten Bedenken hinsichtlich ihrer Verwaltungskapazitäten. Letztlich und ironischer Weise musste Estland seinen internationalen Handel deliberalisieren und Zolltarife einführen.

Politisch stellte die Frage nach dem Umgang mit der russischen Minderheit den schwierigsten und sensibelsten Bereich der Beitrittsverhandlungen dar. Die Hauptfragen bezogen sich auf die Staatsbürgerschaft und Sprache. Da die baltischen Staaten rechtlich als wiederhergestellte Staaten und nicht als Nachfolgestaaten der Sowjetunion anerkannt wurden, er-

4 Kyllike Sillaste-Elling: The Path to Receiving an invitation for Accession Negotiations - the Critical Years of 1996-1997, in: Estonian Ministry of Foreign Affairs (Hrsg.): Estonia's Way into the European Union, Tallinn 2009, S. 23-29, hier S. 26.

5 North Atlantic Treaty Organization.

6 Der Warschauer Pakt hatte folgende Mitglieder: Sowjetunion, Albanien (bis 1968; die Mitgliedschaft ruhte bereits seit 1962), Polen, Ungarn, die Deutsche Demokratische Republik, die Tschechoslowakei und Bulgarien.

7 Klaudijus Maniokas/Ramūnas Vilipišauskas/Darius Žeruolis (Hrsg.): Lithuania's Road to the European Union: Unification of Europe and Lithuania's EU Accession Negotiation, Vilnius 2005, S. 297-349. 
hielten nicht alle Einwohner automatisch deren Staatsbürgerschaft. Vielmehr mussten diejenigen, die sich während der sowjetischen Zeit auf dem Territorium der drei Staaten niedergelassen hatten, die Einbürgerung beantragen. Als wichtigste Bedingung für die Verleihung der Staatsbürgerschaft galten Grundkenntnisse der Landessprache. Aber anstatt einen estnischen oder lettischen Sprachtest abzulegen, entschieden sich viele für die russische Staatsbürgerschaft und eine große Anzahl blieb staatenlos. Eine Mission der Organisation für Sicherheit und Zusammenarbeit in Europa (OSZE) überwachte in Tallinn und Riga ab 1993 den Umgang mit der russischsprachigen Minderheit. Nachdem ein Großteil der OSZEEmpfehlungen von der estnischen und der lettischen Regierung umgesetzt worden war, wurde die Mission 2001 abgeschlossen. ${ }^{8}$

Litauen hatte keine große russische Minderheit und gewährte daher allen Einwohnern die Staatsbürgerschaft. Die einzige nennenswerte ethnische Minderheit in Litauen sind Polen, aber dies stellte kein Problem während der Beitrittsverhandlungen dar. Polen und Litauen schafften es, ihre Differenzen mit der Unterzeichnung eines Freundschaftvertrags im Jahr 1994 beizulegen, was auf beiden Seiten auch durch den Wunsch motiviert war, alle potenziellen Hindernisse auf dem Weg zur Integration in die euro-atlantischen Strukturen zu beseitigen.

Problematisch war (und ist) für Litauen Kaliningrad, eine russische Exklave, die immer noch die am stärksten militarisierte Region in Europa ist. Im Jahr 2002 dominierte die Kaliningrad-Frage kurzzeitig die Agenda der Beziehungen zwischen der Europäischen Union und Russland. Der russische Präsident Wladimir Putin verlangte, dass die Einwohner Kaliningrads frei und ohne Visum über Litauen in das russische Mutterland reisen dürfen. Die Europäische Union weigerte sich jedoch damals, Ausnahmen hinsichtlich des künftig auf die baltischen Staaten auszuweitenden Schengen-Regimes zu machen. Schließlich wurde mit der Schaffung von speziellen, vereinfachten Visa für den Transit ein Kompromiss gefunden. ${ }^{9}$ Zuletzt sorgte Kaliningrad wieder für Beunruhigung, da Russland angeblich taktische Atomraketen in die Exklave verlegt hat. ${ }^{10}$

Zusammen mit Polen, Ungarn, der Tschechischen Republik, der Slowakei, Slowenien, Zypern und Malta schlossen die baltischen Staaten erfolgreich ihre EU-Beitrittsverhandlungen auf dem Kopenhagener Gipfel im Dezember 2002 ab. Es ist zwar richtig, dass die Konditionalitätspolitik der Europäischen Union und die Umsetzung des acquis communautaire den Reformprozess in den baltischen Staaten vorangetrieben hat, den Esten, Letten und Litauern wurde aber auch bewusst, dass diese Reformen wichtig und notwendig für ihre eigenen Länder waren. Die Rechtsvorschriften der Sowjet-Ära mussten auf jeden Fall modernisiert werden und es war einfacher, dem acquis communautaire als Vorbild zu folgen als neues Recht praktisch aus dem Nichts zu entwerfen.

Der EU-Beitritt wurde insbesondere von der Elite unterstützt und es gab eine offenkundige Differenz zwischen den Meinungen der Elite und der Bevölkerung. In Estland und Lettland war die Gruppe der Skeptiker des EU-Beitritts im Vergleich zu den mittelosteuropäischen Ländern am größten. Die litauische Bevölkerung war hingegen begeistert und Litauen gehörte zu den ersten Staaten, die ein Mitgliedschaftsreferendum im Jahr 2003 abhielten, in dem 90 Prozent dem Beitritt zustimmten. Die estnische und lettische Regierung, auf der

8 Pater Van Elsuwege: From Soviet Republics to EU Member States. A Legal and Political Assessment of the Baltic States' Accession to the EU, Leiden 2008, S. 286-287.

9 Kestutis Paulaukas: The Baltics: from nation states to member states, European Union Institute for Security Studies: Occasional Paper Nr. 62, S. 15-16.

10 Der Spiegel: Keine Atomraketen in Kaliningrad - behauptet Gouverneur Jegorow, 11/2001, S. 152. 
anderen Seite, verzögerten aus taktischen Gründen die Referenden bis die anderen Kandidatenländer für den Beitritt gestimmt hatten, um so ein positives Votum zu befördern. Die baltischen Staaten wurden schließlich am 1. Mai 2004 Mitglieder der Europäischen Union.

Es ist wichtig, sich in Erinnerung zu rufen, dass die Beitrittsverhandlungen parallel zu den Verhandlungen über einen NATO-Beitritt stattfanden. Auch wenn beide Beitrittsverfahren offiziell nicht miteinander in Verbindung standen, so beeinflussten sich die Ereignisse doch gegenseitig. Für Litauen, Lettland und Estland war die NATO-Mitgliedschaft noch verlockender als die EU-Mitgliedschaft. Sicherheit war die oberste Priorität aller drei Staaten, da ihre jüngste Vergangenheit durch deren Fehlen gekennzeichnet war. Die Europäische Union wurde zu diesem Zeitpunkt in erster Linie als Binnenmarkt mit fehlender Sicherheitsdimension wahrgenommen. So erschien der EU-Beitritt als realistisch, während die NATO-Mitgliedschaft der baltischen Staaten keine ausgemachte Sache war. Von Seiten Russlands und vieler westlicher Meinungsmacher gab es hiergegen starke Einwände.

\section{Die neuen politischen Systeme: Erfolgsmodelle aus dem Ausland und staatliches Erbe}

Beim Aufbau neuer Institutionen nahmen sich die Esten, Letten und Litauer insbesondere Erfolgsmodelle aus Deutschland und den nordischen Ländern zum Vorbild. Sie griffen aber auch auf einige Elemente aus der Zeit ihrer Unabhängigkeit in den 1920er Jahren zurück. Alle drei baltischen Staaten haben ein parlamentarisches Einkammersystem. In Estland und Lettland werden die Abgeordneten nach Parteilisten mit einem Verhältniswahlsystem gewählt, Litauen hingegen hat ein Mischsystem aus Mehrheits- und Verhältniswahl ähnlich dem personalisierten Verhältniswahlrecht in Deutschland. Der estnische und lettische Präsident werden vom Parlament gewählt und haben vor allem symbolische Macht. In Litauen besitzt der Präsident mehr Machtbefugnisse, teilweise auch weil er direkt gewählt wird.

Seit ihrer Unabhängigkeit sind in Estland und Lettland fast nur Mitte-Rechts-Koalitionen an der Macht gewesen, obwohl sich kein stabiles Parteiensystem gebildet hat. Diese Instabilität wird durch die Tatsache deutlich, dass bis 2006 jede Parlamentswahl in Lettland von einer politischen Partei gewonnen wurde, die bei der vorhergehenden Wahl noch nicht existierte.

Da sich die Nachfolgepartei der litauischen kommunistischen Partei erfolgreich in eine europäische sozialdemokratische Partei transformiert hat, hat Litauen ein völlig anderes Parteiensystem. Ein Grund für diese Transformation ist, dass in Litauen die kommunistische Partei eine wichtige Rolle bei der Erreichung der Unabhängigkeit gespielt hat, während die kommunistischen Parteien in Estland und Lettland von ethnischen Russen dominiert waren und somit als Fremdkörper wahrgenommen wurden.

Anders als in Lettland und Estland wechselte die Regierungsmacht in Litauen hauptsächlich zwischen zwei großen Blöcken - dem linken (Sozialdemokraten) und dem rechten (Konservative). ${ }^{11}$ Ein Ausnahmefall der jüngsten politischen Geschichte Litauens stellt die Amtsenthebung des Außenseiters und populistischen Präsidenten Rolandas Paksas im Jahr 2004 dar, nachdem er die Staatsbürgerschaft einem seiner wichtigsten Geldgeber für die Wahlkampagne gewährt hatte - einem russischen Waffenhändler.

11 See Vello Pettai/Daunis Auers/Ainė Ramonaite: Political Development, in: Marju Lauristin (Hrsg.): Estonian Human Development Report: Baltic Way(s) of Human Development: Twenty Years On, Tallinn 2011, S. $144-165$ 


\section{Die baltischen Staaten als EU-Mitglieder}

\section{Die heutige Politik: hin zu mehr Stabilität}

Nach dem EU-Beitritt traten die heftigsten politischen Turbulenzen in Lettland auf. Da ,Oligarchen' die politischen Parteien finanzierten, häuften sich die Korruptionsvorwürfe in der lettischen Politik. Die öffentliche Entrüstung erreichte 2011 ihren Höhepunkt als der aus dem Amt scheidende Präsident Valdis Zatlers die Auflösung des Parlaments anstrengte. In dem obligatorischen Referendum über die Frage der Parlamentsauflösung, erhielt der Antrag Zatlers große Zustimmung und das Parlament wurde aufgelöst. ${ }^{12}$

Im Gegensatz zu den Übergangsjahren, die von kurzlebigen Regierungen geprägt waren, hat Estland heute eine der stabilsten Regierungen in Europa. Der estnische Premierminister Andrus Ansip, der bereits seit April 2005 im Amt ist, ist derzeit der dienstälteste Regierungschef in der Europäischen Union. ${ }^{13}$

Angesichts der zahlreichen Regierungswechsel infolge der Umsetzung von Sparmaßnahmen zur Überwindung der gegenwärtigen Krise ist es erstaunlich, dass sowohl der estnische Premierminister Ansip als auch der lettische Premierminister Valdis Dombrovskis nach den Parlamentswahlen 2011 wieder zum Regierungschef gewählt wurden, obwohl beide Sparmaßnahmen erlassen hatten. Eine eher beunruhigende Entwicklung in den Hauptstädten Riga und Tallinn ist die wachsende Macht von Nils Ušakovs Partei Harmoniezentrum beziehungsweise Edgar Savisaars Zentrumspartei. Beide Parteien, die hauptsächlich durch russische Wähler unterstützt werden, haben sich zu den größten nationalen Parteien entwickelt und bauen systematisch ein alternatives Machtzentrum als Gegengewicht zur Regierung auf.

In Litauen wurde die konservative Regierung von Andrius Kublius, die eine erfolgreiche Sparpolitik durchsetzte, nach den Wahlen 2012 von den Sozialdemokraten im Amt abgelöst. Es ist allerdings besorgniserregend, dass auch populistische Parteien jeder Couleur in Litauen sehr erfolgreich waren. Zwei von ihnen sitzen nun als Juniorpartner in der Koalitionsregierung (die Arbeiterpartei und die Partei Ordnung und Gerechtigkeit, die vom ehemaligen Präsidenten Paksas gegründet wurde).

Die Integration ethnischer Minderheiten bleibt weiterhin eine Herausforderung und hat sich keineswegs mit der EU-Mitgliedschaft erledigt. Estland wurde mit dem Konflikt um den „Bronzenen Soldaten“ im Jahr 2007 daran erinnert, als russische Jugendliche gegen die von der Regierung beschlossene Versetzung des sowjetischen Soldatenehrenmals aus dem Zentrum von Tallinn protestierten. In Lettland wurden 2011 genügend Unterschriften gesammelt, um über die Frage, ob Russisch die zweite offizielle Landessprache werden soll, eine Volksabstimmung durchzuführen. Dies wurde jedoch von einer Dreiviertelmehrheit abgelehnt. In Litauen zog die Frage nach den Rechten der polnischen Minderheit, insbesondere was den Schulunterricht in der Muttersprache anbetrifft, im Jahr 2010 heftige Wortgefechte zwischen der polnischen und litauischen Regierung nach sich. Seit der Aufnahme der polnischen Minderheitenpartei in die Regierungskoalition im November 2012 hat sich der bilaterale Streit etwas beruhigt.

Paradoxer Weise scheint die europäische Integration die gesellschaftliche Integration der Minderheiten in den baltischen Staaten zu hemmen. Seit dem Beitritt der baltischen Staaten

12 Juris Dreifelds: Latvia, in: Freedomhouse (Hrsg.): Nations in Transit 2012: Democratization from Central Europe to Eurasia, Washington/New York 2013, S. 321-323.

13 Wenn man von dem noch amtierenden Premierminister Luxemburgs, Jean-Claude Juncker, absieht, der aller Voraussicht nach in Kürze aus dem Amt scheiden wird. 
zum Schengen-Raum haben die staatenlosen Russen wenig Anreiz, die Staatsbürgerschaft in Estland oder Lettland zu beantragen. Als Personen mit ständigem Wohnsitz dürfen sie fast genauso frei in der Europäischen Union reisen und arbeiten wie Unionsbürger, zugleich können sie aber mit der russischen Staatsbürgerschaft ihre familiären und geschäftlichen Beziehungen zu Russland pflegen, da Russland kein Visum verlangt und es ihnen erlaubt ist, dort Eigentum zu besitzen. Obwohl sie normativ unterprivilegiert erscheinen, genießen sie praktisch das Beste aus beiden Welten.

\section{EU-Reform: Plädoyer für die Gemeinschaftsmethode}

Während des Konvents zur Zukunft Europas und der Ausarbeitung des Vertrags über eine Verfassung für Europa umwarb das Vereinigte Königreich die baltischen Staaten, vor allem Estland, emsig, um eine tiefere Integration, insbesondere in den Bereichen der Sozial- und Steuerpolitik sowie der Sicherheits- und Verteidigungspolitik, zu verhindern. Aufgrund ihrer offenen, liberalen Volkswirtschaften mit niedrigen Einheitssteuersätzen, denen sie die erfolgreiche Überwindung des sowjetischen Erbes der Planwirtschaft zuschrieben, waren die baltischen Staaten von der Perspektive einer stärkeren EU-Harmonisierung in diesem Bereich alarmiert. Als frische NATO-Mitglieder misstrauten sie EU-Strukturen im Bereich der Sicherheits- und Verteidigungspolitik, die die transatlantischen Beziehungen duplizieren oder gar unterhöhlen könnten. Darüber hinaus war die nationale Souveränität für die drei jungen Staaten, die ihre Unabhängigkeit gerade erst wiedererlangt und hart erkämpft hatten, von besonderer Bedeutung. Somit waren die baltischen Staaten zunächst geneigt, dem intergouvernementalen Modell europäischer Integration eher zuzustimmen als der Gemeinschaftsmethode.

Gleichwohl hatten sie während des Beitrittsprozesses gelernt, dass die Europäische Kommission der , beste Freund ' kleiner neuer Mitglieder ist, weil sie sicherstellt, dass die kleinen Mitgliedstaaten fair behandelt und nicht von großen übergangen werden. Dementsprechend haben die baltischen Staaten bisher keinen Vorschlägen zugestimmt, die die Befugnisse der Kommission geschmälert hätten. Während Estland und Lettland zurückhaltend waren, was die weitere Vertiefung der Europäischen Union betrifft, demonstrierte Litauen seinen Eifer und ratifizierte im Jahr 2004 als erster Mitgliedstaat den Verfassungsvertrag. ${ }^{14}$

Ein Wendepunkt für das Verständnis der baltischen Staaten von der Dynamik der Europäischen Union war die Unterzeichnung des Nord-Stream-Vertrags zwischen dem damaligen deutschen Bundeskanzler Gerhard Schröder und dem russischen Präsidenten Putin im Jahr 2005. Der Bau der Unterwasser-Pipeline in der Ostsee stand in eklatantem Widerspruch zu den baltischen Interessen. Dies schockierte die Balten so sehr, dass sie die Europäische Union aufriefen, mit einer Stimme gegenüber Russland aufzutreten, die Gemeinsame Sicherheitsund Außenpolitik zu stärken und eine gemeinsame Energiepolitik zu schaffen. Dieses Ereignis hat die Balten von der Bedeutung der Gemeinschaftsmethode im Allgemeinen überzeugt.

Bei den Verhandlungen zum Vertrag von Lissabon zeigten alle drei baltischen Staaten eine größere Begeisterung für eine tiefere Integration als je zuvor, sodass die Ratifizierung des Vertrags kaum zu Debatten führte. Eine der größten Ängste der baltischen Staaten war, dass ein Zwei-Klassen-Europa geschaffen werden könnte, bei dem sie nicht zu den Kernstaaten gehören. Die Erweiterung des Schengen-Raums um die baltischen Staaten im De-

14 Andres Kasekamp/ViljarVeebel: Overcoming doubts: The Baltic States and the European Security and Defence Policy, in: Andres Kasekamp (Hrsg.): The Estonian Foreign Policy Yearbook 2007, Tallinn 2007, S. 11-12. 
zember 2007 war ein wichtiger Schritt für sie, um diese Selbstwahrnehmung als Mitgliedstaaten ,zweiter Klasse` zu überwinden.

Estland ist als erster der drei baltischen Staaten bereits im Jahr 2011 der Eurozone beigetreten. Die Aussicht auf den Beitritt stellte im Vorfeld einen positiven Anreiz für Haushaltskürzungen dar und wendete die Gefahr einer forcierten Abwertung der estnischen Währung ab. Damit war der Beitritt ein Weg, um aus der finanziellen Krise zu kommen. Die Mitgliedschaft in der Eurozone konfrontiert Estland jedoch inzwischen mit einer neuen unerwarteten Verantwortung: Das Land muss Solidarität in Form von Krediten für Mitgliedstaaten der Eurozone zeigen, die die Regeln missachtet haben, die Estland strikt eingehalten hat. Die Unterzeichnung des Vertrags über den Europäischen Stabilitätsmechanismus (ESM) löste die erste ernsthafte öffentliche Debatte in Estland über die Europäische Union seit dem Beitritt des Landes 2004 aus. Initiator war der Justizkanzler, ${ }^{15}$ der beim obersten Gericht (Riigikohus) Beschwerde einlegte, da der ESM die estnische Verfassung verletze. Dieses entschied jedoch knapp zugunsten der Regierung, was den Weg für die Ratifizierung des ESM-Vertrags durch das estnische Parlament am 30. August 2012 frei machte. Um die Opposition zu besänftigen, erweiterte die Regierung die Befugnisse des Parlaments, in Zukunft bei ESM-bezogenen Entscheidungen stärker mitwirken zu können.

Am 1. Januar 2014 wird Lettland als zweites der baltischen Länder der Eurozone beitreten, nachdem die Europäische Kommission und die Eurogruppe am 5. Juni 2013 zu dem Schluss kamen, dass auch Lettland die Konvergenzkriterien erfüllt. Die Mehrheit der lettischen Bevölkerung ist derzeit jedoch gegen die Einführung des Euro, da sie Preissteigerungen befürchtet sowie die mögliche Perspektive, reichere Mitglieder der Eurozone retten zu müssen.

Dann wird allein Litauen nicht Mitglied der Eurozone sein, das in seinem Bemühen zu den Kernländern der Europäischen Union zu gehören, bereits 2007 versucht hatte der Eurozone beizutreten. Litauens Antrag wurde damals abgelehnt, weil die Inflationsrate knapp über den Konvergenzkriterien (nur 0,1 Prozent über dem Grenzwert) lag. Im Nachhinein ist dies nicht überraschend, da sich die Wirtschaft der ,baltischen Tiger' überhitzt hatte. 2013 verkündete die litauische Regierung, im Frühjahr 2014 einen neuen Antrag auf Beitritt zur Eurozone zu stellen, in der Hoffnung 2015 beitreten zu können.

\section{Baltische Politik und Prioritäten auf EU-Ebene}

Auf EU-Ebene setzen sich Estland, Lettland und Litauen für eine Stärkung der Sicherheit im Internet, Energiesicherheit, die Östliche Partnerschaft, die Liberalisierung des Energiemarkts, Transparenz, Innovation und die Vollendung des Binnenmarkts (insbesondere im Bereich des Dienstleistungsverkehrs) ein und sind überzeugte Befürworter der EU-Erweiterung. Auch die Zusammenarbeit zwischen den baltischen und den nordischen Ländern als eine regionale Einheit innerhalb der Europäischen Union wird weiter vorangetrieben. Neue Hoffnungen für eine solche regionale Annäherung brachte die von der Europäischen Union ausgearbeitete Ostseestrategie aus dem Jahr 2009, die aber über kein eigenes Budget verfügt. Die nordisch-baltische Koordination gilt innerhalb der Europäischen Union als vielseitig und nutzbringend. ${ }^{16}$

Die baltischen Staaten haben natürlich auch Nischenprojekte, die sie versuchen, auf die EU-Agenda zu setzen. Insbesondere Litauen ist besorgt um die Energiesicherheit und einer

15 Der Justizkanzler in Estland ist in seiner Funktion einem Ombudsmann vergleichbar, hat darüber hinaus aber auch die Aufgabe, die Einhaltung der Verfassung durch die politischen Institutionen zu überwachen.

16 Bernd Henningsen/Tobias Etzold/Anna-Lena Pohl (Hrsg.): Political State of the Region Report 2013: Trends and Directions in the Baltic Sea Region, Kopenhagen 2013. 
der Befürworter der eigentumsrechtlichen Entflechtung von Energieunternehmen im Rahmen des dritten Energiebinnenmarktpakets. Ein Ziel der baltischen Staaten ist, ihre Abhängigkeit von Russland zu beenden, in dem sie, gefördert von EU-Fonds, ihre Infrastrukturen mit jenen im Herzen Europas verbinden. Eine der Prioritäten für Estland ist das sehr ambitionierte Bahnprojekt, welches Tallinn mit Warschau verbinden soll. Darüber hinaus setzt sich Estland für die Förderung der digitalen Wirtschaft und der Computer- und Internetsicherheit ein. Ein Leuchtturmprojekt der estnischen Europapolitik für die Jahre 2011 bis 2015 ist die Schaffung eines digitalen Binnenmarkts. Aus Estland stammt das Unternehmen für Internettelefonie „Skype“ und die Regierung ist im Bereich des E-Government besonders fortschrittlich. Estland ist das erste Land, das eine nationale Parlamentswahl online durchführte. Die Schaffung eines digitalen EU-Binnenmarkts gehört auch zu den Prioritäten der litauischen Ratspräsidentschaft. ${ }^{17}$

Von Beginn an waren die Balten enthusiastische Unterstützer der Europäischen Nachbarschaftspolitik und fanden hier schnell ihre Nische. ${ }^{18}$ Mit der von Polen und Schweden initiierten Östlichen Partnerschaft, ${ }^{19}$ deren Gründungsgipfel 2009 stattfand, gewann diese an Schwung. Für Litauen war die Beteiligung von Belarus ein Anliegen besonderer Wichtigkeit. Im Jahr 2009 versuchte die litauische Präsidentin Dalia Grybauskaitė, entgegen dem europäischen Mainstream, Belarus stärker einzubinden. Diese Politik scheiterte jedoch als das Regime des belarussischen Präsidenten Alexander Lukaschenko im Dezember 2010 brutal gegen die oppositionellen Präsidentschaftskandidaten vorging. Erfolgreicher war Grybauskaitė allerdings im Umgang mit dem ukrainischen Präsidenten Viktor Janukowitsch als sie darauf bestand, die inhaftierte Oppositionsführerin Julia Timoschenko im Krankenhaus zu besuchen.

Der Beitritt zur Europäischen Union bedeutete auch, dass die baltischen Staaten nicht mehr zu den Empfängern, sondern zu den Gebern von EU-Hilfe gehören. Sie versuchen aktiv, ihr Wissen und ihre Erfahrungen aus dem eigenen Reform- und Beitrittsprozess an die osteuropäischen Länder weiterzugeben, die beim Zusammenbruch der Sowjetunion zwei Dekaden vorher mit ihnen in der gleichen Ausgangssituation waren, jedoch bisher nicht die gleichen Fortschritte erzielt haben. ${ }^{20}$ Die wichtigsten Zielländer der estnischen, lettischen und litauischen Entwicklungszusammenarbeit sind Georgien, Moldau und die Ukraine sowie Afghanistan, wo alle drei Länder Soldaten stationiert haben. Hier zeigt sich eine Überschneidung von Werten und geopolitischen Interessen. Insbesondere in Bezug auf ehemalige Sowjetrepubliken sind die baltischen Staaten in der einmaligen Position, die sachkundigsten und effektivsten Geberländer zu sein.

Die baltischen Staaten zeigten sich zufrieden mit dem neuen mehrjährigen Finanzplan 2014 bis 2020, der auf dem Europäischen Rat im Februar 2013 vereinbart wurde. ${ }^{21}$ Während der Haushaltsverhandlungen richtete sich die öffentliche Aufmerksamkeit im Baltikum be-

17 Programm des litauischen Vorsitzes im Rat der Europäischen Union. 1. Juli - 31. Dezember 2013. Für ein Glaubwürdiges, Wachsendes und offenes Europa, S. 7, abrufbar unter: http://static.eu2013.1t/uploads/docume nts/Presidency_Programme_DE.pdf (letzter Zugriff: 14.11.2013).

18 David J. Galbreath/Ainius Lasas/Jeremy W. Lamoreaux: Continuity and Change in the Baltic Sea Region. Comparing Foreign Policies, Amsterdam/New York 2008, S. 127.

19 Katrin Böttger: Im Osten nichts Neues? Ziele, Inhalte und Ergebnisse der Östlichen Partnerschaft, in: integration 4/2009, S. 372-387.

20 Evelin Andrespok/Andres Kasekamp: Development Cooperation of the Baltic States: A Comparison of the Trajectories of Three New Donor Countries, in: Perspectives on European Politics and Society 1/2012, S. 117-130.

21 Europäischer Rat: Europäischer Rat Tagung vom 7./8. Februar 2013. Schlussfolgerungen (Mehrjähriger Finanzrahmen), EUCO 37/13. 
sonders auf zwei Aspekte: die fortdauernde Ungleichbehandlung baltischer Landwirte gegenüber den Landwirten in den alten Mitgliedstaaten und die von der Kommission vorgeschlagene Fazilität „Connecting Europe“ ${ }^{\text {22 }}$, von der sich die baltischen Staaten eine Finanzierung für die Baltische Bahn sowie für einen regionalen Flüssiggasterminal erhoffen.

Erwähnenswert ist, dass ein baltischer Staat bisher nur einmal von seinem Vetorecht in der Europäischen Union Gebrauch gemacht hat und das war Litauen im Jahr 2008 als es das Verhandlungsmandat für ein neues EU-Partnerschafts- und Kooperationsabkommen mit Russland blockierte. ${ }^{23}$ Obwohl sich später zeigte, dass die Sorge Litauens über Russlands Doppelspiel im schwelenden Konflikt in Abchasien berechtigt war, wurde Litauen zunächst für seine Sturheit kritisiert und sogar Estland und Lettland stellten sich auf die Seite der Mehrheit innerhalb der Europäischen Union.

Alle drei baltischen Staaten schafften es, sich neue Agenturen der Europäischen Union in ihre Hauptstädte zu holen. Litauen machte 2007 den Anfang mit dem Europäischen Institut für Gleichstellungsfragen. Das Gremium Europäischer Regulierungsstellen für elektronische Kommunikation wurde in Riga angesiedelt und in 2012 hat schließlich die Europäische Agentur für das Betriebsmanagement von IT-Großsystemen im Raum der Freiheit, der Sicherheit und des Rechts ihre Arbeit in Tallinn aufgenommen.

\section{Ausblick}

\section{Allgemeine Zukunftstrends}

Nachdem die baltischen Staaten drastische Sparmaßnahmen erlassen hatten, sind sie die am schnellsten wachsenden Volkswirtschaften in der Europäischen Union geworden. Estland ist das jüngste Mitglied der Eurozone und Lettland wird 2014 als nächstes Land beitreten, gefolgt von Litauen voraussichtlich im Jahr 2015. Dies ist ein Zeichen dafür, dass die baltischen Staaten weiterhin eine Zugehörigkeit zum europäischen Kern anstreben. Tatsächlich ist Estland das am stärksten integrierte Land Nordeuropas gemessen an der Mitgliedschaft in internationalen Organisationen: Europäische Union ${ }^{24}, \mathrm{NATO}^{25}, \mathrm{OECD}^{26}$ und Eurozone ${ }^{27}$.

Eine große politische Herausforderung für die baltischen Staaten ist ihre Bevölkerungsstruktur, die Bevölkerung nimmt beständig ab und ist überaltert. Die baltischen Staaten verfolgen eine sehr restriktive Einwanderungspolitik als Reaktion auf das sowjetische Erbe. Die Arbeitslosenquote ist niedrig, bedingt durch eine massenhafte Auswanderung von Arbeitskräften in andere EU-Länder. Dieser Trend begann bereits in den boomenden Jahren Mitte der 2000er Jahre als Großbritannien und Irland als einzige EU-Staaten keine Beschränkungen gegen den Zuzug von Bürgern aus den neuen Mitgliedstaaten erließen und so zu einem beliebten Ziel baltischer Auswanderer wurden. Hunderttausende haben Litauen und Lettland verlassen, aus Estland dagegen ist die Abwanderung mit einigen Zehntausenden viel gerin-

22 Europäische Kommission: Vorschlag für eine Verordnung des Europäischen Parlaments und des Rates zur Schaffung der Fazilität „Connecting Europe“, KOM (2011) 665.

23 Ramūnas Vilpišauskas: Lithuanian foreign policy since EU accession: Torn between history and interdependence, in: Michael Baun/Dan Marek (Hrsg.): The New Member States and the European Union. Foreign Policy and Europeanization, Abingdon/New York 2013, S. 127-141, hier S. 139.

24 Island und Norwegen sind keine Mitgliedstaaten der Europäischen Union.

25 Finnland und Schweden sind keine Mitgliedstaaten der NATO.

26 Lettland und Litauen sind keine Mitgliedstaaten der Organisation for Economic Co-operation and Development.

27 Dänemark, Litauen und Schweden (sowie Island und Norwegen) haben nicht den Euro eingeführt. 
ger. Wichtigstes Zielland der estnischen Auswanderer ist das benachbarte Finnland, sodass viele von ihnen zwischen finnischer Arbeitsstätte und estnischem Wohnort pendeln. ${ }^{28}$

\section{Die litauische Ratspräsidentschaft}

Seit dem 1. Juli 2013 ist Litauen der erste baltische Staat, der die rotierende EU-Ratspräsidentschaft inne hat. Litauens Auftreten wird von Lettland und Estland genau beobachtet, um Erfahrungen für ihre eigene Ratspräsidentschaft im ersten Halbjahr 2015 beziehungsweise im ersten Halbjahr 2018 zu sammeln. Das Motto der litauischen Ratspräsidentschaft lautet: „Für ein glaubwürdiges, wachsendes und offenes Europa“. Unter Glaubwürdigkeit fällt das Ziel, Europa finanziell zu stabilisieren und die Bankenunion zu schaffen. Wachstum umfasst die Ziele, den Binnenmarkt zu vertiefen und stärker zu integrieren. Mit dem Wort offen bezieht sich die Ratspräsidentschaft auf die globale Rolle der Europäischen Union, insbesondere in Bezug auf die Nachbarschaft. Eine der wichtigsten Aufgaben, die die litauische Ratspräsidentschaft von der vorhergehenden irischen übernommen hat, ist der Abschluss der Verhandlungen über den nächsten mehrjährigen Finanzrahmen für die Jahre 2014 bis 2020. Dieser wurde schließlich im November 2013 erreicht. Eines der wahrscheinlich am meisten erwarteten Ereignisse ist das Gipfeltreffen der Östlichen Partnerschaft im November 2013 in Vilnius. Die Hoffnung aller drei baltischen Staaten ist, dass die Länder der Östlichen Partnerschaft der Europäischen Union stärker angenähert werden. Das größte Ziel, das Litauen während seiner Ratspräsidentschaft in diesem Politikfeld erreichen möchte, ist die Unterzeichnung von Assoziationsabkommen mit der Ukraine und/oder der Republik Moldau und Georgien.

Die EU-Ratspräsidentschaft stellt eine große Herausforderung für die litauische Regierung dar, die erst seit November 2012 im Amt ist und deren Mitglieder wenig Regierungserfahrung sowie kaum englische Sprachkenntnisse mitbringen. Erfreulicherweise ist der Außenminister Linas Linkevičius, der eine wichtige Rolle bei der Durchführung der Präsidentschaft hat, sehr erfahren und kompetent. Darüber hinaus spielt auch die litauische Präsidentin Grybauskaitė, ehemalige Europäische Kommissarin für Finanzplanung und Haushalt sowie Trägerin des Karlspreises 2013, eine zentrale Rolle während der EU-Präsidentschaft. In Litauen verfügt die Präsidentin im Vergleich zu den Staatsoberhäuptern rein parlamentarischer Regierungssysteme über mehr Kompetenzen, wenn diese auch nicht so umfassend wie in präsidentiellen Regierungssystemen, wie in Frankreich, sind. Ihr Wirken im Europäischen Rat wie auch das des EU-Vorsitzes Litauens insgesamt dürfte sich nicht nur für die baltischen Staaten als eine lohnenswerte Analyse für 2014 anbieten!

Übersetzung aus dem Englischen: Katja Treichel.

28 Aldis Purs: Baltic Facades. Estonia, Latvia and Lithuania since 1945, London 2012, S. 179. 